\title{
SPECTRAL ANALYSIS OF BIRTH-AND-DEATH PROCESSES WITH AND WITHOUT KILLING VIA PERTURBATION*
}

\author{
MORITZ SIMON $^{\dagger}$ AND GALLIANO VALENT ${ }^{\ddagger}$
}

\begin{abstract}
A population process with constant birth rates $\lambda_{n} \equiv \lambda$ and death rates $\mu_{n} \equiv \mu$ is supplied with strong linear killing rates $\gamma_{n}=\gamma n$ for $n \in \mathbb{N}_{0}$. The process is analyzed in view of its spectral representation: The underlying orthogonal polynomials are seen to be Lommel polynomials $R_{n, \nu}(x)$, considered as functions in their parameter $\nu$. Regarding the Stieltjes transform of their orthogonality measure, we recognize that it is purely discrete and given by the zeros of a Bessel function $J_{\nu-1}(x)$ in its order. Qualitative and quantitative results for the zeros are worked out via methods from the theory of Bessel functions and from regular perturbation theory. The same ideas are applied successfully to study the spectrum for associated Meixner polynomials, corresponding to linear birth and death rates.
\end{abstract}

Key words. Birth-and-death processes with killing, spectral representation, Stieltjes transform, Lommel polynomials, Bessel functions, regular perturbation theory, associated Meixner polynomials.

AMS subject classifications. Primary 33C45, 60J80; Secondary 33C10, 47A55

1. Introduction: New killing structures. The study of birth-and-death processes with killing was initiated by S. Karlin and S. Tavaré in [8]. A general treatment of such structures from a spectral point-of-view has recently been worked out by E. van Doorn and A. Zeifman in [16]: Given birth rates $\lambda_{n}$, death rates $\mu_{n}$ and killing rates $\gamma_{n}$ at any state $n \in \mathbb{N}_{0}$, the transition probabilities of the process $\mathcal{X}$ after time $t \geq 0$ may be expressed as

$$
\begin{gathered}
\mathbb{P}\left(\mathcal{X}\left(t+t_{0}\right)=m \mid \mathcal{X}\left(t_{0}\right)=n\right) \equiv \mathbb{P}_{n m}(t)=\frac{1}{\pi_{n}} \int_{0}^{\infty} e^{-x t} F_{n}(x) F_{m}(x) \mathrm{d} \pi(x) \\
\forall n, m \in \mathbb{N}_{0} .
\end{gathered}
$$

Here $\left\{F_{n} \mid n \in \mathbb{N}_{0}\right\}$ is a set of orthogonal polynomials (OP) with a corresponding probability measure $\pi$ supported on $[0, \infty)$. They satisfy the recurrence relation

$$
\mu_{n+1} F_{n+1}(x)+\lambda_{n-1} F_{n-1}(x)=\left(\lambda_{n}+\mu_{n}+\gamma_{n}-x\right) F_{n}(x) \quad \forall n \in \mathbb{N}_{0},
$$

initialized by $F_{0}=1$ and $F_{-1}=0$, while the coefficients $\pi_{n}$ are explicitly given as $\pi_{n}=\prod_{k=1}^{n} \frac{\lambda_{k-1}}{\mu_{k}}$ for $n \in \mathbb{N}_{0}$. Despite this somewhat easy access, up to now there have only been two (nontrivial) processes with killing whose spectral representation could be obtained explicitly:

1. The linear process with killing, having rates $\lambda_{n}=\lambda(n+b), \mu_{n}=\mu n$ and $\gamma_{n}=\gamma n$ for $n \in \mathbb{N}_{0}$, was solved in [8]; but its solution leads back to the well-known Meixner polynomials.

2. The special quadratic process with rate structure $\lambda_{n}=2(n+1)(2 n+1)$, $\mu_{n}=2 n(2 n+1)$ and $\gamma_{n}=2 a(2 n+1)^{2}$ was considered in [5]; it leads to new OP of an elliptic-like character.

\footnotetext{
${ }^{*}$ Received September 1, 2008; accepted for publication January 20, 2009.

$\dagger$ Technische Universität München, Zentrum Mathematik, Boltzmannstraße 3, D-85747 Garching, Germany (moritz.simon@gmail.com).

${ }^{\ddagger}$ Laboratoire de Physique Théorique et des Hautes Energies, CNRS, Unité associée URA 280, 2 Place Jussieu, F-75251 Paris Cedex 5, France; and Département de Mathémathiques, UFR SciencesLuminy, Case 901, 163 Avenue de Luminy, F-13258 Marseille Cedex 9, France.
} 
An extension of the linear process towards associated Meixner polynomials actually just adding an emigration rate while the killing is dropped - will be considered towards the end of the article. We find it interesting to start from the pure immigration-emigration process with constant rates $\lambda_{n} \equiv \lambda$ and $\mu_{n} \equiv \mu$. Within this framework, there are three different types of killing to study:

- One might consider weak killing, for instance with reciprocal rates $\gamma_{n}=\frac{b}{n+a}$ for $n \in \mathbb{N}_{0}$. Anyway, in the latter situation we are only led back to Pollaczek's OP.

- Another possibility would be a process with asymptotically constant killing, for instance supplied with $q$-linear rates $\gamma_{n}=c\left(1-q^{n}\right)$ where $q \in(0,1)$. The polynomials arising here were thoroughly described by M.E.H. IsMAIL and F. Mulla in [4].

- Finally, one could encounter strong killing - the easiest example consists in linear rates $\gamma_{n}=\gamma n$. Quadratic or higher powers of $n$ could also be considered, with increasing difficulty.

The primary aim of our article is the discussion of that third problem, where we focus on linear killing. The main surprise is that the corresponding OP are the Lommel polynomials $R_{n, \nu}(x)$, considered as OP in their parameter $\nu$; their spectral measure happens to be purely discrete, as was first recognized by D. MAKI via chain sequence methods in [11].

The paper is organized as follows: In Section 2 we detect the OP in the spectral representation (1)(2) of the birth-and-death process with killing under the aforementioned rate structure

$$
\lambda_{n} \equiv \lambda, \quad \mu_{n} \equiv \mu \quad \text { and } \quad \gamma_{n}=\gamma n \quad \forall n \in \mathbb{N}_{0} .
$$

In Section 3 we introduce the Stieltjes transform of their orthogonality measure, which appears to be purely discrete. In Section 4 we discuss results of J. CoulomB on the location of the mass points, which we improve in several respects. Use of KatoRellich theory allows to establish the uniform convergence of perturbation series in Section 5, while detailed perturbative formulas are determined in Section 6. Sections 7 through 9 are devoted to the same problems for the discrete spectrum of associated Meixner polynomials, for which little was known so far; the convergence results are more limited here.

2. Underlying Lommel polynomials. We consider the rates (3) under the natural positivity constraints $\lambda, \mu, \gamma>0$. The notational change

$$
F_{n}(x) \equiv\left(\frac{\lambda}{\mu}\right)^{\frac{n}{2}} \mathcal{F}_{n}(\nu ; \rho) \quad \forall n \in \mathbb{N}_{0} \quad \text { where } \quad \nu:=\frac{\lambda+\mu-x}{\gamma}, \quad \rho:=\frac{\sqrt{\lambda \mu}}{\gamma}
$$

brings the three-term recurrence relation (2) for the OP to the more tractable form

$$
\begin{aligned}
& (\nu+n) \mathcal{F}_{n}(\nu ; \rho)=\rho\left[\mathcal{F}_{n+1}(\nu ; \rho)+\mathcal{F}_{n-1}(\nu ; \rho)\right] \quad \forall n \in \mathbb{N}, \\
& \mathcal{F}_{0}(\nu ; \rho)=1, \quad \mathcal{F}_{1}(\nu ; \rho)=\frac{\nu}{\rho} .
\end{aligned}
$$

Since $\gamma \rightarrow 0$ for $\varrho \rightarrow \infty$, i. e. the killing vanishes, we must recover Chebyshev polynomials in that limit: Indeed the limiting relations are explicitly given by

$$
\lim _{\rho \rightarrow \infty} \mathcal{F}_{n}(\rho x ; \rho)=T_{n}(x) \quad \text { and } \quad \lim _{\rho \rightarrow \infty} \mathcal{F}_{n}(2 \rho x ; \rho)=U_{n}(x) \quad \forall n \in \mathbb{N}_{0}
$$


Thereby the polynomials $\mathcal{F}_{n}$ appear as generalizations of the Chebyshev polynomials of first and second kind. Comparing (5) with (2) on p. 299 of [17], we then recognize that the OP under consideration are nothing but Lommel polynomials - the crucial relation

$$
F_{n}(x) \equiv\left(\frac{\lambda}{\mu}\right)^{\frac{n}{2}} \mathcal{F}_{n}(\nu ; \rho)=\left(\frac{\lambda}{\mu}\right)^{\frac{n}{2}} R_{n, \nu}(2 \rho) \quad \forall n \in \mathbb{N}_{0}
$$

classifies the birth-death polynomials through the definitions in (4). There remains the question about the orthogonality measure $\psi$ of those polynomials. Anyway, for the moment let us make some remarks on the nature of the Lommel polynomials under consideration:

1. The functions $\mathcal{F}_{n}(\nu ; \rho)$ are in fact polynomials of degree $n$ both in the variable $\nu$ and in their inverse parameter $\rho^{-1}$. This follows easily from

$$
\mathcal{F}_{n}(\nu ; \rho)=R_{n, \nu}(2 \rho)=\sum_{k=0}^{\left\lfloor\frac{n}{2}\right\rfloor}(-1)^{k}\left(\begin{array}{c}
n-k \\
k
\end{array}\right) \rho^{2 k-n}(\nu+k)_{n-2 k} \quad \forall n \in \mathbb{N}_{0},
$$

which is a direct consequence of relation (3) on p. 296 in [17].

2. The functions $R_{n, \nu}(x)$ satisfy a fourth-order ODE in their variable $x \equiv 2 \rho$; see p. 297 in [17].

3. The Lommel polynomials are of hypergeometric type, since they can also be written as

$$
\mathcal{F}_{n}(\nu ; \rho)=R_{n, \nu}(2 \rho)=\frac{(\nu)_{n}}{\rho^{n}}{ }_{2} F_{3}\left(\begin{array}{c}
-\frac{n}{2},-\frac{n+1}{2} \\
\nu,-n, 1-n-\nu
\end{array} \mid-4 \rho^{2}\right) \quad \forall n \in \mathbb{N}_{0} ;
$$

compare (5) on p. 297 of [17]. In a similar way, the relation

$$
\mathcal{G}_{n}(\nu ; \rho)=\frac{(\nu+1)_{n}}{\rho^{n}}{ }_{2} F_{3}\left(\begin{array}{c|c}
\frac{1-n}{2},-\frac{n}{2} \\
\nu+1,1-n, 1-n-\nu
\end{array} \mid-4 \rho^{2}\right) \quad \forall n \in \mathbb{N}_{0}
$$

expresses the "numerator polynomials" $\mathcal{G}_{n}(\nu ; \rho):=R_{n, \nu}^{*}(2 \rho)$ in hypergeometric form.

4. With $x \equiv 2 \rho$ the three-term recurrence relation (5) may be rewritten as

$$
R_{n+1, \nu}(x)+R_{n-1, \nu}(x)=\frac{2(n+\nu)}{x} R_{n, \nu}(x) \quad \forall n \in \mathbb{N},
$$

which is nothing but recursion (2) on p. 299 of [17]. This relation demonstrates a different manner of showing the polynomial character both in $\nu$ and in $x^{-1}$. The Lommel polynomials $R_{n, \nu}(x)$ are therefore not really polynomials in their variable $x$, but only in its inverse $x^{-1}$.

5. Due to (6) the Lommel polynomials generalize Chebyshev polynomials of first and second kind in the following sense: $R_{n, \rho x}(2 \rho) \rightarrow T_{n}(x)$ and $R_{n, 2 \rho x}(2 \rho) \rightarrow$ $U_{n}(x)$ as $\rho \rightarrow \infty$.

3. Asymptotics and Stieltjes transform. Hurwitz' limit relation on p. 302 of [17] allows to derive the asymptotics

$$
\mathcal{F}_{n}(\nu ; \rho) \sim \frac{\Gamma(n+\nu)}{\rho^{n+\nu-1}} J_{\nu-1}(2 \rho), \quad \mathcal{G}_{n}(\nu ; \rho) \sim \frac{\Gamma(n+\nu)}{\rho^{n+\nu}} J_{\nu}(2 \rho) \quad \forall \nu \in \mathbb{C} \backslash \mathbb{R}
$$


as $n \rightarrow \infty$ for the OP and their numerator polynomials. Now uniqueness of the corresponding spectral measure $\psi$ may be guaranteed as follows - other methods also work.

Proposition 1. The Hamburger moment problem corresponding to the OP $\mathcal{F}_{n}(\nu ; \rho)$ from $(5)$ is determinate.

Proof. Let us first observe that the polynomials $\mathcal{F}_{n}(\nu ; \rho)$ are orthonormal, which follows from their recurrence (5). Theorem 2.9 in [13] then asserts that the divergence of the series $\sum_{n=0}^{\infty}\left|\mathcal{F}_{n}(\nu ; \rho)\right|^{2}$ for one complex $\nu \in \mathbb{C} \backslash \mathbb{R}$ is sufficient for the determinacy of the Hamburger moment problem. Take $\nu=i$ : Coulomb's results from the next section show that $J_{i-1}(2 \rho)$ cannot vanish. By virtue of the polynomial asymptotics (7), we thereby have $\left|\mathcal{F}_{n}(i ; \rho)\right|^{2} \sim$ const $\cdot \rho^{2(1-n)}|\Gamma(n+i)|^{2}$ with const $\equiv\left|J_{i-1}(2 \rho)\right|^{2}>0$, which in turn is readily seen to diverge as $n \rightarrow \infty$ upon use of Stirling's formula.

As the moment problem is determinate, one may use (7) together with Markov's theorem [15] to get the Stieltjes transform of the probability measure $\psi$ for the OP $\mathcal{F}_{n}(\nu ; \rho)$, which is already known [17].

Proposition 2. The Stieltjes transform $\Psi$ of the orthogonality measure $\psi$ for the $O P \mathcal{F}_{n}(\nu ; \rho)$ is given by

$$
\Psi(\nu):=\int_{\mathbb{R}} \frac{\mathrm{d} \psi(t)}{\nu-t} \stackrel{!}{=} \lim _{n \rightarrow \infty} \frac{\mathcal{G}_{n}(\nu ; \rho)}{\mathcal{F}_{n}(\nu ; \rho)}=\frac{\rho^{-\nu} J_{\nu}(2 \rho)}{\rho^{1-\nu} J_{\nu-1}(2 \rho)} \quad \forall \nu \in \mathbb{C} \backslash \mathbb{R},
$$

which is a ratio of two entire (modified) Bessel functions. This special shape implies that for arbitrary values $\rho>0$ the spectrum $\operatorname{supp}(\psi)$ must be discrete, purely consisting of isolated mass points.

It is interesting to note the following continued fraction expansion of the Stieltjes transform:

$$
\rho \Psi(\nu)=\frac{J_{\nu}(2 \rho)}{J_{\nu-1}(2 \rho)} \equiv \underline{1} / \frac{\nu}{\rho}-\underline{1} / \frac{\nu+1}{\rho}-\underline{1} / \frac{\nu+2}{\rho}-\ldots
$$

The finite form of the continued fraction (8) is given on p. 153 of [17]; it provides yet another interesting recursive property of Bessel functions, namely

$$
\frac{J_{\nu}(2 \rho)}{J_{\nu-1}(2 \rho)} \equiv \underline{1} / \frac{\nu}{\rho}-\underline{1} / \frac{\nu+1}{\rho}-\underline{1} / \frac{\nu+2}{\rho}-\ldots-\underline{1} / \frac{\nu+m}{\rho}-\frac{J_{\nu+m+1}(2 \rho)}{J_{\nu+m}(2 \rho)} \quad \forall m \in \mathbb{N} .
$$

It has been noted in [11] that the above continued fraction was already illustrating the orthogonality of the Lommel polynomials in the variable $\nu$, being the order of the associated Bessel functions. Therein D. MAKI mainly utilized some general methods to show that the spectrum in $\nu$ must be discrete, not paying attention to the fact that it indeed consists of the zeros of an entire function, which immediately implies his conclusion. We shall investigate this remarkable property more closely during the following sections. Notice also that the Lommel polynomials $R_{n, \nu}(x)$ are orthogonal in the inverse variable $x^{-1}$, while the orthogonality measure is purely discrete and given by the inverse zeros of the Bessel function $J_{\nu-1}(x)$ in its variable; this was first mentioned in the rather general setting of [3]. 
4. General spectral results. Proposition 2 demonstrates that the mass points $\widetilde{x}_{n}$ in the spectrum $\operatorname{supp}(\psi):=\left\{\widetilde{x}_{n} \mid n \in \mathbb{N}_{0}\right\}$ of our population process correspond to zeros $\nu_{n}$ of the Bessel function $J_{\nu-1}(2 \rho)$ for $\rho>0$ via the relation $\widetilde{x}_{n}=\lambda+\mu-\gamma \nu_{n}$ for $n \in \mathbb{N}_{0}$. Their equation can be written as

$$
\rho^{1-\nu} J_{\nu-1}(2 \rho) \equiv \mathcal{J}_{\nu-1}(r)=\sum_{k=0}^{\infty} \frac{(-r)^{k}}{k ! \Gamma(k+\nu)}=0 \quad \text { where } \quad r=\rho^{2}
$$

It shows that the true parameter of the problem is $r=\rho^{2}$. Regarding the roots $\nu_{n}(r)$ as functions of the positive variable $r$, the following results have been proved in [2]:

Theorem [Coulomb]. For every $r>0$ the roots $\nu_{n}(r)$ of equation (9) are real, simple and asymptotic to the negative integers $-n$ as $n \rightarrow \infty$. The functions $\nu_{n}:(0, \infty) \rightarrow \mathbb{R}$ are continuous and increasing.

Notice here that the entire functions $\mathcal{J}$ from (9) satisfy the symmetry relation $\mathcal{J}_{-p}(r)=(-1)^{p} \mathcal{J}_{p}(r)$ for $p \in \mathbb{N}_{0}$ and $r \in \mathbb{C}$. An easy consequence can be derived as follows.

Proposition 3. Let us denote by $r_{n}$ for $n \in \mathbb{N}$ the well-known zeros of $\mathcal{J}_{1}(r)$. Then, given that $r \in\left(r_{n}, r_{n+1}\right)$ for some $n \in \mathbb{N}$, the order zeros $\nu_{0}(r)>\ldots>\nu_{n}(r)$ will be strictly positive, the rest being strictly negative.

Proof. In the limiting case $r=0$, the zeros correspond to poles of the gamma function $\Gamma(\nu)$, which are $\nu_{n}(0)=-n$ for $n \in \mathbb{N}_{0}$. From Coulomb's results we now that the $\nu_{n}(r)$ are continuously increasing with $r>0$. So, when $r$ starts increasing from zero, the largest zero $\nu_{0}(r)$ must always be strictly positive. Let us then consider the second zero $\nu_{1}(r)$ : it starts from $\nu_{1}(0)=-1$ and increases. It will reach zero when $\mathcal{J}_{-1} \equiv-\mathcal{J}_{1}$ will vanish, that is for $r=r_{1}$; so for $r \in\left(0, r_{1}\right)$ we will only have one strictly positive mass, namely $\nu_{0}(r)$. For $r>r_{1}$ we will have two strictly positive masses $\nu_{0}(r)$ and $\nu_{1}(r)$ up to $r=r_{2}$, where a third positive mass $\nu_{2}(r)$ must accordingly appear. Recursively we get the stated criterion.

Zeros of the Bessel function in its variable have already been determined to high accuracy (see e.g. [17]); the numerical values of the first few $r_{n}$ are therefore given by

$$
r_{1} \approx 3.67049, \quad r_{2} \approx 12.30461, \quad r_{3} \approx 25.87486, \quad r_{4} \approx 44.38019, \quad r_{5} \approx 67.82041 .
$$

Notice that only a finite number of positive zeros is possible from Proposition 3 . This agrees with Horn's uniform asymptotic expansion from p. 225 of [17], explicitly

$$
J_{\nu}(2 \rho)=e^{\nu\left(1-\log \frac{\nu}{\rho}\right)-\frac{1}{2} \log \nu}\left[\frac{1}{\sqrt{2 \pi}}+\mathcal{O}\left(\nu^{-1}\right)\right] \quad \text { for } \quad \nu \gg \rho,
$$

which also shows that $J_{\nu}(2 \rho)$ as a function of $\nu>0$ is eventually positive; apparently the same holds for the shifted function $J_{\nu-1}(2 \rho)$. Let us next prove some bounds for the spectrum.

Proposition 4. Given small $r \in\left(0, r_{1}\right)$, we have the uniform inclusion $\nu_{n}(r) \in$ $(-n, 1-n)$ for all $n \in \mathbb{N}_{0}$; here again $r_{1} \approx 3.67049$ denotes the initial zero of our deformed Bessel function $\mathcal{J}_{1}$. 
Proof. Remember that the functions $\nu_{n}(r)$ for $n \in \mathbb{N}_{0}$ are the zeros of

$$
\rho^{1-\nu} J_{\nu-1}(2 \rho) \equiv \mathcal{J}_{\nu-1}(r)=\sum_{k \geq 0} \frac{(-r)^{k}}{k ! \Gamma(k+\nu)},
$$

which is an entire function of $\nu \in \mathbb{C}$. Let us rather consider the function

$$
H(\nu ; r):=\Gamma(\nu) \mathcal{J}_{\nu-1}(r)=1+\sum_{k=1}^{\infty} \frac{(-r)^{k}}{k !(\nu)_{k}} .
$$

It is meromorphic with simple poles $-n$ for $n \in \mathbb{N}_{0}$, induced by the Gamma function; but since the Gamma function never vanishes, both functions $H(\nu ; r)$ and $\mathcal{J}_{\nu-1}(r)$ have exactly the same zeros. Let us now insert into (10) the partial fraction expansion

$$
\frac{1}{(\nu)_{k}}=\sum_{p=1}^{k} \frac{(-1)^{p-1}}{(p-1) !(k-p) !} \frac{1}{\nu+p-1} \quad \forall k \in \mathbb{N},
$$

and interchange the order of the summations to receive the new writing

$$
H(\nu ; r)=1+\sum_{p=1}^{\infty} \frac{(-1)^{p-1}}{(p-1) !(\nu+p-1)} \sum_{k=p}^{\infty} \frac{(-r)^{k}}{k !(k-p) !} .
$$

Using the notation defined in (9), due to symmetry we first have

$$
H(\nu ; r)=1-\sum_{p=1}^{\infty} \frac{\mathcal{J}_{p}(r)}{(p-1) !(\nu+p-1)} ;
$$

the crucial Bessel-theoretic identity

$$
\sum_{p=0}^{\infty} \frac{\mathcal{J}_{p}(r)}{p !} \equiv 1 \quad \forall r \in \mathbb{C}
$$

then allows us to express the function $H$ in the uniform sum

$$
H(\nu ; r)=\sum_{p=0}^{\infty} \frac{\mathcal{J}_{p}(r)}{p !} \frac{\nu-1}{\nu+p-1} .
$$

Let us now take the variable $\nu$ to be real. The derivative of $H$ off the poles is merely

$$
H^{\prime}(\nu ; r)=\sum_{p=1}^{\infty} \frac{\mathcal{J}_{p}(r)}{(p-1) !} \frac{1}{(\nu+p-1)^{2}},
$$

and for $r \in\left(0, r_{1}\right)$ all Bessel terms $\mathcal{J}_{p}(r)$ for $p \in \mathbb{N}$ are strictly positive; hence the derivative is strictly positive and $H(\nu ; r)$ is strictly increasing on any open interval of its real definition range. For any fixed $r \in\left(0, r_{1}\right)$ this implies (intermediate value theorem!) that there is a respective unique zero $\nu_{n}(r)$ in each interval $(-n, 1-n)$ for $n \in \mathbb{N}$, while the largest zero satisfies $\nu_{0}(r) \in(0,1)$ according to the mentioned identity (11), which after all necessitates the evaluation $H(1 ; r)=1$. 
5. Convergent perturbation theory: Kato-Rellich. Before we delve into perturbative computations, let us first see under which condition on $r=\rho^{2}$ absolute convergence of the "Rayleigh-Schrödinger series" $\nu_{n} \equiv \nu_{n}(r)$ is assured. Denote by $\left\{\mathfrak{e}_{n} \mid n \in \mathbb{N}_{0}\right\}$ the canonical basis of the Hilbert space $\ell^{2}\left(\mathbb{N}_{0}\right)$. Then the recurrence (5) of the $\mathrm{OP} \mathcal{F}_{n}(\nu ; \rho)$ corresponds to a self-adjoint Jacobi operator $H \equiv H(\rho)$, working in $\ell^{2}\left(\mathbb{N}_{0}\right)$ through its action on the basis

$$
H \mathfrak{e}_{n}=\rho \mathfrak{e}_{n+1}-n \mathfrak{e}_{n}+\rho \mathfrak{e}_{n-1} \quad \forall n \in \mathbb{N}_{0},
$$

while we interpret $\mathfrak{e}_{-1} \equiv 0$. Apparently, $H$ can be understood as a combination $H(\rho) \equiv H_{0}+\rho V$ of the two operators

$$
H_{0} \mathfrak{e}_{n}:=-n \mathfrak{e}_{n} \quad \text { and } \quad V \mathfrak{e}_{n}:=\mathfrak{e}_{n+1}+\mathfrak{e}_{n-1} \quad \forall n \in \mathbb{N}_{0} .
$$

Here $H_{0}$ is an unbounded diagonal operator with pure point spectrum $\nu_{n}(0) \equiv-n$ for $n \in \mathbb{N}_{0}$, which is perturbed by the bounded operator $V$. In the terminology of [12], the perturbation $V$ is relatively compact, hence the perturbed operator $H(\rho)$ has compact resolvent for all $\rho \in \mathbb{C}$. Since $V$ is a bounded operator, the combination $H(\rho)=H_{0}+\rho V$ constitutes a so-called analytic operator family, being self-adjoint for $\rho \in \mathbb{R}$. Theorem 3.9 in Chapter 7 of [9] thereby implies that the spectrum of $H(\rho)$ is still a pure point spectrum, only consisting of analytic perturbations $\nu_{n}(\rho)$ of the eigenvalues $\nu_{n}(0)=-n$. The following question remains: When are the perturbed eigenvalues $\nu_{n}(\rho)$, which are of course nothing but the zeros of $J_{\nu-1}(2 \rho)$, expressible in terms of their perturbation series, which are nothing but the Rayleigh-Schrödinger series from general perturbation theory? The upcoming auxiliary result is due to T. Kato and F. Rellich; see e. g. Theorems XII.8/11 in [12].

Lemma [Kato-Rellich]. Let $H_{0}$ and $V$ be operators in some Hilbert space with common domain $\mathcal{D}$ and assume $\|V \phi\| \leq A\left\|H_{0} \phi\right\|+B\|\phi\|$ holds for $\phi \in \mathcal{D}$, where $A, B \geq 0$ are suitable constants. Moreover suppose $H_{0}$ be self-adjoint with nondegenerate isolated eigenvalues $\nu_{n}(0)$ for $n \in \mathbb{N}_{0}$. Denote

$$
\delta_{n}:=\operatorname{dist}\left(\nu_{n}(0), \sigma\left(H_{0}\right)\right) \quad \text { and } \quad \rho_{n}:=\frac{\delta_{n}}{2 A\left(\left|\nu_{n}(0)\right|+\delta_{n}\right)+2 B} \quad \forall n \in \mathbb{N}_{0} .
$$

Then the corresponding perturbed eigenvalues $\nu_{n}(\rho)$ of the analytic operator family $H(\rho)=H_{0}+\rho V$ are analytic functions in the respective discs $|\rho|<\rho_{n}$ for $n \in \mathbb{N}_{0}$, where their Rayleigh-Schrödinger perturbation series (i.e. their Taylor-McLaurin expansions!) converge absolutely.

In Chapter 5 of the $\mathrm{PhD}$ thesis [14], the above Kato-Rellich lemma has been exploited to determine convergence ranges for killing-dominated birth-and-death processes: there a diagonal killing operator is perturbed by a tridiagonal birthdeath operator. Especially, given polynomial rates as in our case, Theorem 5.5 of [14] gives some applicable convergence criteria. In our situation (13) the killing polynomial is linear, strictly dominating the constant birth and death rates. Since $H_{0}$ is diagonal here, the perturbation series for shifted operators $H+a \equiv H_{0}+a+\rho V$ with arbitrary $a \geq 0$ converge in identical ranges. This freedom in the choice of the shift $a$ can be utilized to prove that the perturbation series $\nu_{n} \equiv \nu_{n}(r)$ constitute entire functions in $r=\rho^{2}$, since the diagonal domination is strict — being of higher degree than the tridiagonal perturbation (see the proof of Theorem 5.5 in [14]).

Proposition 5. The Taylor-McLaurin expansions - i. e. Rayleigh-Schrödinger perturbation series - of the zeros $\nu_{n} \equiv \nu_{n}(r)$ converge absolutely for $r \in \mathbb{C}$, which makes the extended functions $\nu_{n}: \mathbb{C} \rightarrow \mathbb{C}$ entire. 
6. Some perturbative computations. This section is devoted to perturbative computations of the zeros $\nu_{n}=\nu_{n}(r)$ for $n \in \mathbb{N}_{0}$. According to Proposition 5, the convergence of the involved series expansions happens for any complex $r=\rho^{2} \in \mathbb{C}$; for values $r \equiv \frac{\lambda \mu}{\gamma^{2}}>0$ the model corresponds to an immigration-emigration process with linear killing under no further restrictions. In any case, the roots $\nu \equiv \nu_{n}$ of (9) are to be found.

In the marginal case $r=0$, the order zeros of the Bessel function evidently correspond to poles of the gamma function $\Gamma(\nu)$, which are given by $\nu_{n}(0)=-n$ for $n \in \mathbb{N}_{0}$, as we have already noted. Formulas for the first few perturbative corrections (compare Corollary 4.3 and Example 5.8 in [14]) motivate a certain structure for the perturbation. The upcoming result coincides with Theorem 6.7 of [14], but we present its formulation and proof in a slightly different manner, which seems more natural.

Proposition 6. For $r \in \mathbb{C}$ the roots $\nu=\nu_{n}$ of (9) may be expanded as $\nu_{n}(r)=$ $-n+r^{n+1} \sum_{j=0}^{\infty} \mathcal{E}_{n}^{j} r^{j}$, where the successive perturbative corrections $\mathcal{E}_{n}^{j}$ satisfy the recursive relations

$$
\sum_{j=0}^{k} \mathcal{E}_{n}^{j} \frac{(n-k+j) !}{(k-j) !}=\frac{(-1)^{k}}{k !(n+1+k) !} \quad \text { when } \quad k \leq n .
$$

For $k>n$ the complexity of those relations increases drastically, due to additional perturbative terms.

Proof. Fix $n \in \mathbb{N}_{0}$ in what follows; then multiply (9) by $\Gamma(n+\nu+1)$ and split the sum into two separate parts. This leads to the following equivalent relation:

$$
\sum_{k=0}^{n} \frac{(-r)^{k} \Gamma(n+\nu+1)}{k ! \Gamma(k+\nu)}+\sum_{k=n+1}^{\infty} \frac{(-r)^{k} \Gamma(n+\nu+1)}{k ! \Gamma(k+\nu)}=0
$$

Using the convenient notation $\zeta \equiv \nu+n$, we could rewrite the respective gamma ratios as Pochhammer factorials, namely $\frac{\Gamma(n+\nu+1)}{\Gamma(k+\nu)}=(\zeta+k-n)_{n+1-k}$ if $k \leq n$, whereas $\frac{\Gamma(k+\nu)}{\Gamma(n+\nu+1)}=(\zeta+1)_{k-n-1}$ for $k \geq n+1$. Changing the index of the infinite sum to $l=k-n-1$ and multiplying everything by $(-1)^{n+1}$, we can formulate (15) in the rather clear and accessible form

$$
\zeta \sum_{k=0}^{n} \frac{r^{k}(1-\zeta)_{n-k}}{k !}=r^{n+1} \sum_{l=0}^{\infty} \frac{(-r)^{l}}{(n+1+l) !(\zeta+1)_{l}},
$$

which tells us that for the respective zero $\nu_{n} \equiv \zeta-n$ we should receive a perturbative series of the shape $\zeta=r^{n+1} \sum_{k=0}^{\infty} \mathcal{E}_{n}^{j} r^{j}$, where the successive corrections $\mathcal{E}_{n}^{j}$ are to be determined. In order to achieve this, insert the formal perturbative series for $\zeta$ into (16) and divide by $r^{n+1}$ :

$$
\sum_{j=0}^{\infty} \mathcal{E}_{n}^{j} r^{j}\left[\sum_{k=0}^{n} \frac{r^{k}(n-k) !}{k !}+\mathcal{O}\left(r^{n+1}\right)\right]=\sum_{l=0}^{\infty} \frac{(-r)^{l}}{(n+1+l) !}\left[\frac{1}{l !}+\mathcal{O}\left(r^{n+1}\right)\right]
$$

Comparison of the coefficients corresponding to the respective powers of $r$ leads to the wanted recursion (14) for $k \leq n$. For $k>n$ the complexity of the relations increases to some extent, since then the otherwise negligible terms $\mathcal{O}\left(r^{n+1}\right)$ in equation (17) must be involved - this gives further $\mathcal{E}$-terms. $\square$ 

values

Paying attention to those additional $\mathcal{E}$-terms, one gets the explicit numerical

$$
\mathcal{E}_{0}^{1}=-\frac{1}{2} \quad \text { and } \quad \mathcal{E}_{0}^{2}=\frac{7}{12}, \quad \mathcal{E}_{1}^{2}=\frac{15}{16}
$$

for the lowest-order corrections, while the recursion (14) yields the eventual formulas

$$
\begin{aligned}
& \mathcal{E}_{n}^{0}=\frac{1}{n !(n+1) !} \quad \forall n \geq 0, \\
& \mathcal{E}_{n}^{1}=\frac{1}{n !(n+2) !}\left(-2-\frac{2}{n}\right) \quad \forall n \geq 1, \\
& \mathcal{E}_{n}^{2}=\frac{1}{n !(n+3) !}\left[2+\frac{5}{n}-\frac{6}{n^{2}(n-1)}\right] \quad \forall n \geq 2 .
\end{aligned}
$$

We could go to higher orders if needed. One recognition is rather appealing: from the above formulas we get the impression that there should be uniform asymptotics for the corrections as $n \rightarrow \infty$.

Indeed, such asymptotics may be established - in Corollary 6.8 of [14] the investigation was started. However, we are now able to derive more detailed results: The suggested scaling $e_{n}^{j}:=n !(n+j+1) ! \mathcal{E}_{n}^{j}$ of the corrections for $n, j \in \mathbb{N}_{0}$ transforms (14) into the recurrence relation

$$
\sum_{j=0}^{k} \frac{e_{n}^{j}}{(k-j) !} \Pi_{n, k}^{j}=\frac{(-1)^{k}}{k !} \quad \text { where } \quad \Pi_{n, k}^{j}:=\frac{(n+1)_{k+1}}{(n+1+j-k)_{k+1}} .
$$

In the limit $n \rightarrow \infty$, in view of the convergence $\Pi_{n, k}^{j} \rightarrow 1$, the new relation (18) becomes

$$
\sum_{j=0}^{k} \frac{x_{j}}{(k-j) !}=c_{k} \quad \text { where } \quad x_{j}:=\lim _{n \rightarrow \infty} e_{n}^{j}, \quad c_{k}:=\frac{(-1)^{k}}{k !} \quad \forall j, k \in \mathbb{N}_{0} .
$$

Before we come back to (18), let us prove a lemma that is useful for solving such a recursion:

Lemma [G]. For generic values $c_{k}$ equation (19) can be solved for the $x_{j}$, using the generating functions

$$
G(x ; z)=\sum_{j=0}^{\infty} x_{j} z^{j} \quad \text { and } \quad G(c ; z)=\sum_{k=0}^{\infty} c_{k} z^{k}
$$

in view of their functional relation $G(x ; z)=e^{-z} G(c ; z)$. Our values are "generic" in a certain sense!

Proof. Multiply the relation (19) by $z^{k}$ and sum over all $k \in \mathbb{N}_{0}$; this gives the generating function $G(c ; z)$ on the right-hand side. On the left-hand side we reverse the summations

$$
G(c ; z)=\sum_{k=0}^{\infty} z^{k} \sum_{j=0}^{k} \frac{x_{j}}{(k-j) !}=\sum_{j=0}^{\infty} x_{j} z^{j} \sum_{k=j}^{\infty} \frac{z^{k-j}}{(k-j) !}=G(x ; z) e^{z}
$$


to end up with the functional relation. The upcoming proposition clarifies the term "generic".

Proposition 7. For $j \leq n$ the scaled corrections may be expanded into the asymptotic series

$$
e_{n}^{j}=\frac{(-2)^{j}}{j !}\left[1+\frac{j(j+3)}{4 N}+\frac{j(j+3)}{4 N^{2}}+\frac{j(j-1)(j-2)(j+5)}{32 N^{2}}+\mathcal{O}\left(N^{-3}\right)\right]
$$

with $N \equiv n+1$, whereby the "true" corrections are determined through $e_{n}^{j} \equiv n !(n+$ $j+1) ! \mathcal{E}_{n}^{j}$.

Proof. Let us prove that the scaled corrections indeed admit a uniform asymptotic expansion of the form $e_{n}^{j}=\sum_{m=0}^{\infty} \varepsilon_{m}^{j} N^{-m}$ with $N \equiv n+1$. In order to see this, first notice that one certainly has an expansion of the form $\Pi_{n, k}^{j}=1+\sum_{l=1}^{\infty} \gamma_{l, k}^{j} N^{-l}$ with suitable coefficients; the initial coefficients obey

$$
\gamma_{1, k}^{j}=(k+1) K, \quad \gamma_{2, k}^{j}=\gamma_{1, k}^{j}+\frac{1}{2}(k+1)(k+2) K(K-1) \quad \text { where } \quad K \equiv k-j,
$$

but of course more could be computed. Insert both expansions into (18) to have the link

$$
\sum_{j=0}^{n} \frac{\varepsilon_{0}^{j}}{(k-j) !}=\frac{(-1)^{k}}{k !}, \quad \sum_{j=0}^{n} \frac{\varepsilon_{m}^{j}}{(k-j) !}=-\sum_{j=0}^{n} \frac{1}{(k-j) !} \sum_{s+t=m-1} \gamma_{s, k}^{j} \varepsilon_{t}^{j} \quad \forall m \in \mathbb{N}
$$

for any $k \in \mathbb{N}_{0}$. Once we know $\varepsilon_{0}^{j}$ (easy task!), the following $\varepsilon_{m}^{j}$ for $m \in \mathbb{N}$ are uniquely determined. In view of the uniqueness of the solution of (18), we thereby get the asymptotic series of its solution. The remaining task is to compute the generating functions $G\left(\varepsilon_{m} ; z\right) \equiv \sum_{j=0}^{\infty} \varepsilon_{m}^{j} z^{j}$ stepwise, using the strategy of Lemma $[\mathrm{G}]$ in each step. The initial coefficients (21) allow to achieve this for $m \in\{0,1,2\}$ - one gets after straightforward but tedious algebra

$$
G\left(\varepsilon_{0} ; z\right)=e^{-2 z}, \quad G\left(\varepsilon_{1} ; z\right)=z(z-2) e^{-2 z}, \quad G\left(\varepsilon_{2} ; z\right)=G\left(\varepsilon_{1} ; z\right)+\frac{1}{2}(z-4) z^{3} e^{-2 z} .
$$

After expansion in powers of $z$, those generating functions eventually lead to the claim (20).

7. Associated Meixner polynomials. The associated Meixner polynomials correspond to a birth-and-death process without killing, whose birth and death rates are fixed by the relations

$$
\begin{array}{ll}
\lambda_{n}=c(n+\gamma+\beta), & \mu_{n}=n+\gamma\left(1-\eta \delta_{n 0}\right) \quad \forall n \geq 0 \\
\text { where } \quad 0<c<1, & \gamma+\min \{1, \beta\}>0 .
\end{array}
$$

The additional parameter $\eta \in\{0,1\}$ in (22) obeys $\eta=0$ for the standard associated Meixner polynomials, whereas we have $\eta=1$ for their "zero-related" partners. The second case seems to be more adjusted to the modeling of populations because it guarantees $\mu_{0}=0$, which means there can be no emigation when the population has already died out. Anyway, we shall treat both cases.

In both cases $\eta \in\{0,1\}$ of (22), the Stieltjes transform for the measure of the OP was computed in [6]: the spectrum is purely discrete and located at the zeros of the entire function

$$
f(x):=\frac{\Gamma(\eta-x)}{\Gamma(-x) \Gamma(\eta+\gamma-x)}{ }_{2} F_{1}\left(\begin{array}{c}
\gamma, 1-\beta-x \\
\eta+\gamma-x
\end{array} \mid c\right) .
$$


For $\gamma=0$ we recover the Meixner polynomials with the discrete spectrum $n \in \mathbb{N}_{0}$. (For more information about Meixner's OP and their relation to linear birth-anddeath processes with killing let us refer the reader to [8] or Chapter 3 of [14].) However, for $\gamma \neq 0$ very little is known about the spectrum. The ideas developed for the Lommel spectrum will be shown to give some new insights into the associated Meixner spectrum. Let us begin with a result similar to Proposition 4.

Proposition 8. The discrete spectrum for the associated Meixner OP consists of mass points $\widetilde{x}_{n} \equiv \gamma+\eta-\nu_{n}$ with $\nu_{n}>\nu_{n+1}$ for $n \in \mathbb{N}_{0}$, while there appears an additional mass at $\widehat{x}=0$ for $\eta=1$; we set $\nu:=\gamma+\eta-x$. The decreasing numbers $\nu_{n}$ are the roots of the hypergeometric function

$$
\mathbf{F}(\nu):={ }_{2} F_{1}\left(\begin{array}{c}
\gamma, 1-\beta-x \\
\eta+\gamma-x
\end{array} \mid c\right) \equiv \sum_{k=0}^{\infty} \frac{c^{k}(\gamma)_{k}(\nu-\alpha)_{k}}{k !(\nu)_{k}}
$$

while we agree on $\alpha:=\beta+\gamma+\eta-1$. If the two conditions

$$
\min \{\gamma, \alpha\} \geq 0 \quad \text { and } \quad \max \{\gamma, \alpha\} \leq 1
$$

hold simultaneously, then $\nu_{0}>0$ and the other roots obey the inclusion $\nu_{n} \in(-n, 1-n)$ for $n \in \mathbb{N}$.

Proof. The mentioned structure of the spectrum becomes clear from the special shape of the entire function $f$. Now we try to detect the roots $\nu_{n}$ of $\mathbf{F}$. Use of the partial fraction expansion

$$
\frac{(\nu-\alpha)_{k}}{(\nu)_{k}}=\sum_{p=1}^{k} \frac{(-1)^{p-1}(1-p-\alpha)_{k}}{(p-1) !(k-p) !} \frac{1}{\nu+p-1} \quad \forall k \in \mathbb{N}
$$

after reversing the summation order (as in the proof of Proposition 4) leads to

$$
\mathbf{F}(\nu)=1+\sum_{p=1}^{\infty} \frac{(-1)^{p-1}}{(p-1) !(\nu+p-1)} \sum_{k=p}^{\infty} \frac{c^{k}(\gamma)_{k}(1-p-\alpha)_{k}}{k !(k-p) !}
$$

Next change the second summation index to $l=k-p$ and use the factorial facts $(\gamma)_{l+p}=(\gamma)_{p}(\gamma+p)_{l}$ as well as $(1-p-\alpha)_{l+p}=(-1)^{p}(\alpha)_{p}(1-\alpha)_{l}$ to end up with

$$
\mathbf{F}(\nu)=1-\sum_{p=1}^{\infty} \frac{c^{p}(\gamma)_{p}(\alpha)_{p}}{p !(p-1) !} \frac{1}{\nu+p-1}{ }_{2} F_{1}\left(\begin{array}{c|c}
p+\gamma, 1-\alpha \\
p+1
\end{array} \mid c\right),
$$

which off the poles apparently has the derivative

$$
\mathbf{F}^{\prime}(\nu)=\sum_{p=1}^{\infty} \frac{c^{p}(\gamma)_{p}(\alpha)_{p}}{p !(p-1) !} \frac{1}{(\nu+p-1)^{2}}{ }_{2} F_{1}\left(\begin{array}{c}
p+\gamma, 1-\alpha \\
p+1
\end{array} \mid c\right)
$$

Let us now discuss the conditions that ensure the positivity of (24): In order to guarantee positivity in front of the hypergeometric term, we need to fulfill the stricter positivity constraint $\min \{\gamma, \alpha\} \geq 0$, which corresponds to the first condition. (Favard's positivity condition $\min \{\gamma, \alpha\}>-1$ from (22) is weaker!) The hypergeometric term - expanded in its series - becomes positive under the additional constraint $\alpha \leq 1$. 
The alternative $\gamma \leq 1$ can be obtained from a simple Euler transformation of the hypergeometric term. Combining both alternatives, we end up with the second condition $\max \{\gamma, \alpha\} \leq 1$.

Now, given that the derivative (24) is purely positive, the function $\mathbf{F}$ must strictly increase in every interval $(-n, 1-n)$ for $n \in \mathbb{N}$ between the poles. Continuity guarantees unique zeros $\nu_{n} \in(-n, 1-n)$, while the existence of the largest zero $\nu_{0}>0$ follows from (23) in view of $\mathbf{F}(\nu) \rightarrow 1$ as $\nu \rightarrow \infty$. $\square$

8. Kato-Rellich analysis. In order to apply the Kato-Rellich lemma from Section 5, let us interpret the Jacobi operator from (22) as a perturbation $H \equiv H_{0}+\rho V$ of a diagonal operator $H_{0}$, which we may still shift by some $a \geq 0$. This cannot be achieved in choosing the association parameter $\gamma$ for the coupling constant $\rho$. However, it seems that $c$ might be a good choice, since this parameter also represents the variable of the hypergeometric functions at hand. In fact, it is even better to choose $\rho \equiv \sqrt{c}$ as coupling constant: then the operators $H_{0}+a$ and $V$ are given through their action

$$
\left(H_{0}+a\right) \mathfrak{e}_{n} \equiv\left(a+\mu_{n}\right) \mathfrak{e}_{n} \quad \text { and } \quad V \mathfrak{e}_{n} \equiv \alpha_{n} \mathfrak{e}_{n+1}+\rho(n+\gamma+\beta) \mathfrak{e}_{n}+\alpha_{n-1} \mathfrak{e}_{n-1},
$$

where we abbreviate $\alpha_{n}:=-\sqrt{(n+\gamma+\beta) \mu_{n+1}}$ for $n \in \mathbb{N}_{0}$. A suitable choice of the shift $a$ establishes the following result, despite of the fact that $V$ still depends on the parameter $\rho$.

Proposition 9. The perturbation series of the respective mass points $\widetilde{x}_{n} \equiv \gamma+$ $\eta-\nu_{n}(\rho)$ from Proposition 8 converge absolutely for $n \in \mathbb{N}_{0}$ whenever $\rho \equiv \sqrt{c} \in(0,1)$ is chosen such that

$\rho<-1+\sqrt{1+\frac{1}{2(n+\sigma)}}=\frac{1}{4 n}+\mathcal{O}\left(n^{-2}\right) \quad$ with $\quad \sigma:=(1+\gamma)(1+\eta)+\max \{1, \beta\}$.

There is no uniform Kato-Rellich bound under which we could guarantee convergence for all $\nu_{n} \equiv \nu_{n}(\rho)$.

Proof. Choose the shift $a$ large enough, for instance $a:=\gamma \eta+\max \{1, \beta\}$. Some easy arguments using the triangle inequality - compare the proof of Theorem 5.5 in [14] — establish that

$$
\|V \phi\| \leq(2+\rho)\left\|\left(H_{0}+a\right) \phi\right\| \quad \text { whenever } \quad \phi \equiv \sum_{n=0}^{\infty} \phi_{n} \mathfrak{e}_{n} \in \mathcal{D}\left(H_{0}\right)
$$

notice that both $\lambda_{n}$ and $\left|\alpha_{n}\right|$ are dominated by $a+\mu_{n}$ to understand this estimation in fact our shift $a=\gamma \eta+\max \{1, \beta\}$ yields the minimal choice for uniform domination! But this means that one can choose constants $A:=2+\rho$ and $B \equiv 0$ in the sense of the Kato-Rellich lemma. Finally, as the respective unperturbed eigenvalues of $H_{0}+a$ due to (25) are indeed given by $n+\sigma-1$, one has uniform spectral distances $\delta_{n} \equiv 1$. Combination of those recognitions gives bounds

$$
\rho_{n}=\frac{1}{2(2+\rho)(n+\sigma)} \quad \forall n \in \mathbb{N}_{0}
$$

in the Kato-Rellich sense. So the sufficient convergence condition $\rho<\rho_{n}$ yields a quadratic inequality for $\rho \equiv \sqrt{c} \in(0,1)$, whose solution in terms of the coupling constant eventually proves the claim. $\square$ 
One should remark at this point that such Kato-Rellich conditions for convergence are often not at all necessary: Example 5.9 of [14] shows that there may exist a uniform disc of convergence for the spectrum, even if the Kato-Rellich bounds are not uniform; Example 5.10 even allows entire convergence! Nonetheless, it is quite profitable to have sufficient estimations of the convergence ranges.

9. Further perturbative computations. Since hypergeometric series possess a similar structure as the Bessel functions, including Pochhammer factorials thereby Gamma functions - after all, the same ideas as in Section 6 may be used for perturbative computations; we could therefore locate the discrete spectrum as follows:

Proposition 10. In their respective perturbative regimes of Proposition 9, the mass points $\widetilde{x}_{n} \equiv \gamma+\eta-\nu_{n}$ for $n \in \mathbb{N}_{0}$ may be expanded in a perturbation series

$$
\nu_{n} \equiv-n+(\gamma)_{n+1}(\alpha)_{n+1} \mathbf{c}^{n+1} \sum_{j=0}^{\infty} \mathcal{E}_{n}^{j} \mathbf{c}^{j} \quad \text { with } \quad \mathbf{c}:=\frac{c}{1-c}
$$

where the perturbative corrections $\mathcal{E}_{n}^{j}$ are recursively given by

$$
\sum_{j=0}^{k} \mathcal{E}_{n}^{j} \frac{(n-k+j) !(\gamma)_{k-j}(\alpha)_{k-j}}{(k-j) !}=\frac{(-1)^{k}(n+1+\gamma)_{k}(n+1+\alpha)_{k}}{k !(n+1+k) !} \quad \text { when } \quad k \leq n .
$$

Proof. Let us first denote $\zeta=n+\nu$ for fixed $n \in \mathbb{N}_{0}$. A Pfaff transformation of F yields

$$
{ }_{2} F_{1}\left(\begin{array}{c|c}
\gamma, \alpha & -\mathbf{c}
\end{array}\right)=0 \quad \text { with } \quad \nu=\gamma+\eta-x, \quad \alpha=\beta+\gamma+\eta-1, \quad \mathbf{c}:=\frac{c}{1-c} .
$$

All perturbative regimes of Proposition 9 indeed guarantee the necessary condition $|\mathbf{c}|<1$, which we need in order to use the hypergeometric series; now split this series into two pieces and multiply each piece by $(-1)^{n+1}(\nu)_{n+1}$, moreover using some factorial cancelations and the index change $l=k-n-1$ for the infinite sum - in apparent analogy to the proof of Proposition 7. Application of those operations on equation (28) produces the equivalent relation

$$
\zeta \sum_{k=0}^{n} \frac{\mathbf{c}^{k}(\gamma)_{k}(\alpha)_{k}(1-\zeta)_{n-k}}{k !}=(\gamma)_{n+1}(\alpha)_{n+1} \mathbf{c}^{n+1} \sum_{l=0}^{\infty} \frac{(-\mathbf{c})^{l}(n+1+\gamma)_{l}(n+1+\alpha)_{l}}{(n+1+l) !(\zeta+1)_{l}}
$$

which corresponds to (16) from the Lommel proof. The structure of (29) already implies the expanded shape $(26)$ with $\zeta \equiv(\gamma)_{n+1}(\alpha)_{n+1} \mathbf{c}^{n+1} \sum_{j=0}^{\infty} \mathcal{E}_{n}^{j} \mathbf{c}^{j}$. The rest works exactly as in the Lommel case: insert the expansion of $\zeta$ into (29) - this yields a relation similar to (17). Comparison of the coefficients for the respective powers of c then leads to the desired recursion (27); we skip the details. $\mathbf{\square}$

In fact, the situation in Proposition 10 shows a great analogy to Proposition 6. However, the recursion (27) has a more difficult shape this time, since it includes additional factorials in $\alpha$ and $\gamma$. For this reason we could not yet establish as detailed asymptotic treatments for the corrections as in Section 7. Finding a suitable "Lemma [G]" will probably be the hardest task in the associated Meixner setting. 
Acknowledgement. Moritz Simon thanks Rupert Lasser for funding his research visits to Marseille and Aix-en-Provence via $\mathrm{PhD}$ funds from the Helmholtz Zentrum München at the Institute of Biomathemathics and Biometry. Most discussions with Galliano Valent took place during those visits.

\section{REFERENCES}

[1] R. Askey, M.E.H. Ismail, Recurrence relations, continued fractions and orthogonal polynomials, Mem. Am. Math. Soc., 300 (1984), pp. 1-108.

[2] J. Coulomb, Sur les zéros des fonctions de Bessel considérées comme fonction de l'ordre, Bull. Sci. Math., 60 (1936), pp. 297-302.

[3] D. Dickinson, H. Pollack, G. Wannier, On a class of polynomials orthogonal over a denumerable set, Pac. J. Math., 6:2 (1956), pp. 239-247.

[4] M.E.H. Ismail, F. Mulla, On the generalized Chebyshev polynomials, SIAM J. Math. Anal., 18 (1987), pp. 243-258.

[5] M.E.H. Ismail, G. Valent, On a family of orthogonal polynomials related to elliptic functions, Ill. J. Math., 42 (1998), pp. 294-312.

[6] M.E.H. Ismail, J. Letessier, G. Valent, Linear birth and death models and associated Laguerre and Meixner polynomials, J. Approx. Theory, 55 (1988), pp. 337-348.

[7] S. Karlin, J.MCGregor, The differential equations of birth-and-death processes, and the Stieltjes moment problem, Trans. Am. Math. Soc., 85 (1958), pp. 489-546.

[8] S. Karlin, S. Tavaré, Linear birth and death processes with killing, J. Appl. Prob., 19 (1982), pp. $477-487$.

[9] T. Kato, Perturbation Theory for Linear Operators, 2nd ed., corrected, Springer, Berlin, 1995.

[10] H. Koelink, R. Swarttouw, On the zeros of the Hahn-Exton q-Bessel function and associated q-Lommel polynomials, J. Math. Anal. Appl., 186:3 (1994), pp. 690-710.

[11] D. MAKI, On constructing distribution functions - with applications to Lommel polynomials and Bessel functions, Trans. Am. Math. Soc., 130 (1968), pp. 281-297.

[12] M. Reed, B. Simon, Methods of Modern Mathematical Physics IV: Analysis of Operators, Academic Press, San Diego, 1978.

[13] J. Shohat, J. Tamarkin, The Problem of Moments, revised, Am. Math. Soc., Providence, 1950.

[14] M. Simon, Spectral Theory of Birth-and-Death Processes - Explicit Methods with Examples and Perturbative Approaches under Domination of Killing, PhD thesis, Sierke Verlag, Göttingen, 2008.

[15] W. van Assche, Orthogonal polynomials, associated polynomials and functions of the second kind, J. Comp. Appl. Math., 37 (1991), pp. 237-249.

[16] E. Van Doorn, A. Zeifman, Birth-death processes with killing, Stat. Prob. Lett., 72 (2005), pp. 33-42.

[17] G. Watson, Theory of Bessel Functions, 2nd ed., Cambridge University Press, Cambridge, 1980. 